\title{
WEAK LOCALIZATION AND ELECTRON-ELECTRON INTERACTION IN Si/SiGe QUANTUM WELLS*
}

\author{
A. Prinz, G. Stöger, G. Brunthaler, G. Bauer
}

Institut für Halbleiterphysik, Johannes Kepler Universität, $4040 \mathrm{Linz}$, Austria

\section{K. Ismail and B.S. Meyerson}

IBM Thomas J. Watson Research Center, Yorktown Heights New York 10598, USA

A negative magnetoresistance is observed in Si/SiGe modulation doped heterostructures which is attributed to the single particle quantum interference (weak localization) effect. From analysis of the experimental data the electron phase coherence time $\tau_{\phi}$ is extracted to follow a $\left(a T+b T^{2}\right)^{-1}$ dependence. The evaluated prefactor $\alpha=0.25$ is below the theoretical limit of 0.5 , but agrees with observations in $\mathrm{Si}$ and $\mathrm{GaAs} / \mathrm{AlGaAs}$ heterostructures.

PACS numbers: $73.20 . \mathrm{Fz}$

Advances in epitaxial growth techniques of $\mathrm{Si} / \mathrm{Si}_{1-x} \mathrm{Ge}_{x}$ heterostructures have resulted in high-mobility two-dimensional electron (2DEG) and hole gases $[1,2]$. These heterostructures are of considerable importance for possible device applications. Since the carrier mobilities in modulation doped $\mathrm{Si} / \mathrm{Si}_{1-x} \mathrm{Ge}_{x}$ samples available exceed those of Si metal-oxide semiconductor (MOS) devices by far, studies of carrier transport which were previously restricted mainly to the $\mathrm{GaAs} / \mathrm{Ga}_{1-x} \mathrm{Al}_{x}$ As system can now be performed in Si based systems.

Magnetotransport measurements on $\mathrm{Si} / \mathrm{SiGe}$ heterostructures were performed in order to investigate the effects of weak localization (WL) and electron-electron interaction (EEI). Applying the theories of WL for 2D systems, we have extracted from the data the temperature dependence of the phase-breaking time $\tau_{\phi}$ and the prefactor $\alpha$.

The $\mathrm{Si} / \mathrm{Si}_{1-x} \mathrm{Ge}_{x}$ heterostructure $\mathrm{M} 4$ was grown at $550^{\circ} \mathrm{C}$ on high resistivity $p^{-}$substrates using an ultrahigh-vacuum chemical vapor deposition (UHV-CVD) system. A $500 \mathrm{~nm}$ thick $\mathrm{Si} / \mathrm{Si}_{1-x} \mathrm{Ge}_{x}$ superlattice buffer with an average Ge content of 0.3 is grown to serve as a strain relief multi-layer [2]. Above the superlattice (SL) a partly relaxed $50 \mathrm{~nm}$ thick $\mathrm{Si}_{0.7} \mathrm{Ge}_{0.3}$ alloy buffer is grown, followed

*This work is supported by "Fonds zur Förderung der wissensch. Forschung" Austria. 
by the $10 \mathrm{~nm}$ wide tensily strained $\mathrm{Si}$ layer and a $4 \mathrm{~nm}$ wide $\mathrm{Si}_{0.7} \mathrm{Ge}_{0.3}$ spacer. The phosphorus doped $\mathrm{Si}_{0.8} \mathrm{Ge}_{0.2}$ supply layer $\left(N_{\mathrm{D}}=4 \times 10^{18} \mathrm{~cm}^{-3}\right)$ is followed by three cap layers. The biaxially strained Si layer forms the well, in which the sixfold valley degeneracy of the $\mathrm{Si}$ conduction band is removed, and for the two ground state valleys the in-plane effective mass $m_{t}=0.19 m_{0}$ and the vertical mass $m_{l}=0.92 m_{0}$.

The transport measurements were performed on lithographically defined Hall bars with a channel width of $140 \mu \mathrm{m}$, a channel length of $450 \mu \mathrm{m}$ and voltage probes $200 \mu \mathrm{m}$ apart with ohmic AuSb contacts. The magnetoresistance $R_{x x}$ was measured with a $69 \mathrm{~Hz} \mathrm{AC}$ technique and sweeping the magnetic field from negative to positive values without stopping at zero. Hall effect measurements of sample M4 give a carrier concentration $n_{\mathrm{s}}=9.85 \times 10^{15} \mathrm{~m}^{-2}$ and a mobility $\mu=1.53 \mathrm{~m}^{2} /(\mathrm{V} \mathrm{s})$ at $4.2 \mathrm{~K}$.

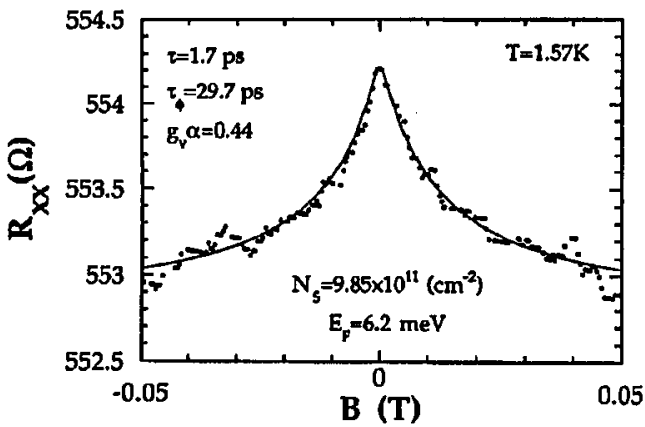

Fig. 1. Magnetoresistance (symbols) for magnetic field perpendicular to the $\mathrm{Si} / \mathrm{SiGe}$ layers at $T=1.57 \mathrm{~K}$ and fitted curve (solid line) as described in the text.

Figure 1 shows the dependence of $R_{x x}$ for small magnetic fields $B$ up to $0.05 \mathrm{~T}$ at $T=1.57 \mathrm{~K}$. The narrow peak (a negative MR) due to the WL effect is clearly visible. Above $B=0.15 \mathrm{~T}$ a positive MR is detected, which is temperature independent and may be caused by a parallel conduction path. For even higher fields above $0.5 \mathrm{~T}$ another negative MR is observed which is strongly temperature dependent and can be attributed to EEI in the diffusion channel [3]. In the following, only the negative MR due to WL below $0.1 \mathrm{~T}$ is analysed in detail.

According to Hikami et al. [4] the effect of WL on the conductivity can be described by

$$
\Delta \sigma_{x x}^{\mathrm{WL}}(B, T)=-\frac{\alpha g_{v} e^{2}}{2 \pi^{2} \hbar}\left[\Psi\left(\frac{1}{2}+\frac{\hbar}{4 e B D \tau_{\mathrm{e}}^{\mathrm{WL}}}\right)-\Psi\left(\frac{1}{2}+\frac{\hbar}{4 e B D \tau_{\phi}(T)}\right)\right]
$$

where $\Psi$ denotes the digamma function with $D-$ the diffusion constant, $\tau_{\mathrm{e}}^{\mathrm{WL}}-$ the elastic scattering time and $\tau_{\phi}$ - the phase coherence time. In the prefactor the valley degeneracy $g_{v}=2$ and a phenomenological factor $\alpha$ enter. In addition to the WL effect, the contribution from the Cooperon is taken into account. The Cooperon describes the electron-electron interaction effect in the particle-particle channel [5]. 
The good agreement between the fitted curve (solid line) and the data points is demonstrated in Fig. 1. For the [it, the elastic scattering time $\tau_{\mathrm{e}}^{\mathrm{WL}}$ was assumed to be equal to the transport time $\tau_{\mathrm{t}}=1.7 \mathrm{ps}$ as calculated from mobility. The obtained phase coherence time $\tau_{\phi}=29.7 \mathrm{ps}$ due to inelastic scattering is much larger than $\tau_{\mathrm{t}}$. A prefactor $\alpha=0.25 \pm 0.03$ is obtained which is smaller than the values between 0.5 and 1 as predicted by theory [6], but is in agreement with estimations of the WL effect from experiments on $\mathrm{Si}$ inversion layers [7] and GaAs/AlGaAs heterostructures [8]. The Cooperon gives a positive MR contribution at small magnetic fields, but according to our fit, this part is smaller than ten per cent of the WL effect. In principle, the weak localization is also affected by spin-orbit scattering but according to fits with the full theoretical expression [9] the spin-orbit scattering time $\tau_{\mathrm{SO}}$ for our samples is at least a factor of 10 larger than $\tau_{\phi}$ and does not contribute.

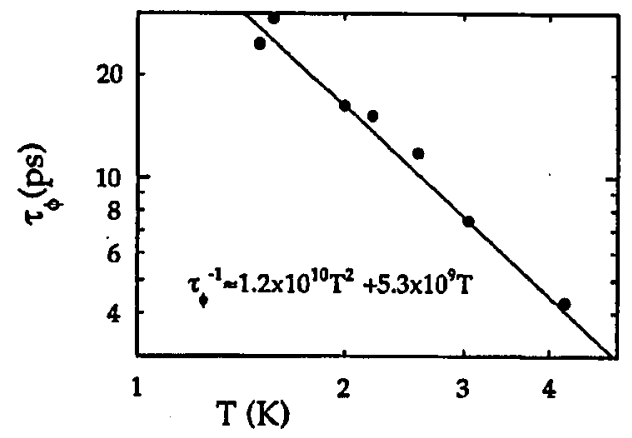

Fig. 2. Values for $\tau_{\phi}$ (symbols) as obtained from fits of the magnetoresistance at different temperatures and a fit of the temperature dependence (solid line) according to $a T+b T^{2}$.

The full circles in Fig. 2 show $\tau_{\phi}$ as obtained from fits of $R_{x x}$ for different $\grave{T}$. At low temperatures the inelastic scattering is dominated by electron-electron interaction and one has to distinguish between the dirty metal and the pure metal case. In the dirty metal case $\tau_{\phi} \propto T^{-1}$ whereas in the pure metal case $\tau_{\phi} \propto T^{-2}[6,10]$. If the system is in an intermediate state, both cases will contribute and $\tau_{\phi}^{-1}$ may be approximated by $a T+b T^{2}$ [11]. The solid line in Fig. 2 represents a fit with $a=5.3 \times 10^{9} \mathrm{~s}^{-1} \mathrm{~K}^{-1}$ and $b=1.2 \times 10^{10} \mathrm{~s}^{-1} \mathrm{~K}^{-2}$ which indicates that our system is in the intermediate regime. The dependence of $\tau_{\phi}$ as deduced from our measurements on a strained Si QW is in good agreement with the theoretical predictions $[6,10,11]$ which give $a^{\prime}=7.09 \times 10^{9} \mathrm{~s}^{-1} \mathrm{~K}^{-1}$ and $b^{\prime}=4.0 \times 10^{9} \mathrm{~s}^{-1} \mathrm{~K}^{-2}$. Therefore the experimentally determined values of $\tau_{\phi}$ are about a factor two smaller than the calculated ones. Essentially similar results were obtained for another $\mathrm{Si} / \mathrm{SiGe}$ modulation doped sample grown on an optimized SiGe alloy buffer layer which allows low temperature mobilities in excess of $10 \mathrm{~m}^{2} /(\mathrm{V} \mathrm{s})$. However, for our investigations, the spacer layer thickness of this sample (M60) was chosen to be just $25 \AA$, and the mobility at $4.2 \mathrm{~K}$ was 
about $0.7 \mathrm{~m}^{2} /(\mathrm{V} \mathrm{s})$. The experiments were performed down to $T=0.35 \mathrm{~K}$, where $\tau_{\phi}$ is about 70 ps. Prasad et al. [12] report for Si/SiGe QW's a dependence of $\tau_{\phi}=L_{\phi}^{2} / D \propto T^{-1.5}$ which also indicates the intermediate regime between dirty and pure metal case.

In summary, the weak localization correction to the conductivity of a $2 \mathrm{D}$ electron gas in a strained $\mathrm{Si} / \mathrm{SiGe}$ heterostructure was evaluated in order to extract the phase coherence time $\tau_{\phi}$ and the prefactor $\alpha$. Concerning $\tau_{\phi}$, our system is in an intermediate range between the pure and dirty metal regime and the evaluated values agree with theory. The prefactor $\alpha(0.25)$ is smaller than its lower limit of 0.5 according to theory [6], but agrees with the analysis of weak localization measurements on $\mathrm{Si}$ inversion layers and $\mathrm{GaAs} / \mathrm{AlGaAs}$ heterostructures.

\section{References}

[1] For a recent review see e.g., Semiconductors and Semimetals, Vol. 33, Ed. T.P. Pearsall, Academic Press, Boston 1993.

[2] K. Ismail, B.S. Meyerson, P.J. Wang, Appl. Phys. Lett. 58, 2117 (1991); K. Ismail, M. Arafa, K.L. Saenger, J.O. Chu, B.S. Meyerson, Appl. Phys. Lett.66, 1077 (1995); Y.H. Xie, E.A. Fitzgerald, D. Monroe, P.J. Silverman, G.P. Watson, J. Appl. Phys. 73, 8364 (1993).

[3] M.A. Paalanen, D.C. Tsui, J.C.M. Hwang, Phys. Rev. Lett. 51, 2226 (1983).

[4] S. Hikami, A.I. Larkin, Y. Nagaoka, Prog. Theor. Phys. 63, 707 (1980).

[5] B.L. Altshuler, A.G. Aronov, A.I. Larkin, D.E. Khmel'nitskii, Sov. Phys. JETP 54, 411 (1981).

[6] S. Kawaji, Prog. Theor. Phys. Suppl. 84, 178 (1985).

[7] M.J. Uren, R.A. Davies, M. Kaveh, M. Pepper, J. Phys. C, Solid State Phys. 14, L395 (1981); D.J. Bishop, R.C. Dynes, D.C. Tsui, Phys. Rev. B 26, 773 (1982); R.A. Davies, M. Pepper, J. Phys. C, Solid State Phys. 16, L353 (1983).

[8] B.J.F. Lin, M.A. Paalanen, A.C. Gossard, D.C. Tsui, Phys. Rev. B 29, 927 (1984).

[9] H. Fukuyama, in: Electron-Electron Interactions in Disordered Systems, Eds. A.L. Efros, M. Pollak, North-Holland, New York 1985, p. 155.

[10] H. Fukuyama, J. Phys. Soc. Jap. 53, 3299 (1984); S. Kawaji, Y. Kawaguchi, J. Phys. Soc. Jap. 53, 2868 (1984).

[11] K.K. Choi, D.C. Tsui, K. Alavi, Phys. Rev. B 36, 7751 (1987).

[12] R. Prasad, T. Thornton, A. Matsumara, D. Williams, in: 22nd Int. Conf. on the Physics of Semiconductors, Ed. D.J. Lockwood, World Scientific, Singapore 1995, p. 1560. 